\title{
Pre-research for Long-term Driving Monitoring System Based on Physiological Signals
}

\author{
Mengxing Liu ${ }^{1,2,3, a}$, Hang Chen ${ }^{1,2,3, b}$ \\ ${ }^{1}$ College of Biomedical Engineering \& Instrument Science, Zhejiang University, China \\ ${ }^{2}$ Key lab of Biomedical Engineering of Ministry of Education, Zhejiang University, China \\ ${ }^{3}$ Zhejiang Provincial Key Laboratory of Cardio-Cerebral Vascular Detection Technology and \\ Medicinal Effectiveness, China \\ amason_liu_bme@163.com, ${ }^{b}$ ch-sun@263.net
}

\begin{abstract}
Keywords: Driving Monitoring, Ballistocardiogram, ECG, Dry contact electrode, Driver fatigue, Piezoelectric film.

Abstract. A novel long-term driving monitoring system with soft conductive foam and piezoelectric polymer film sensors was designed to enable measurement of two physiological signals: the electrocardiogram (ECG) and ballistocardiogram (BCG). The ECG was acquired using dry contact foam electrodes integrated into the steering wheel cover. The BCG was detected using a film type transducer material, which was installed beneath the driver's seat. The experimental results indicated that high- quality signals could be collected and transmitted to on-board computer or personal smart phone while driving at a steady state, except for the duration of one-hand driving operation, acceleration, braking and jolting. This work demonstrates that the unconstrained system can be used for monitoring the physical fatigue and cardiovascular health of people during the period of driving.
\end{abstract}

\section{Introduction}

The World Health Organization (WHO) has reported that about 1.3 million people die each year in road traffic crashes around the world, and the number is expected to double by 2030 unless measures to control them are put in place [1]. Hsing-Chung proved that the two most important factors including not wearing a seat belt and fatigued driving caused severe injuries in crashes of high-deck buses in long-distance driving [2]. It has been suggested that the government should effectively implement education programs to encourage the use of seat belts for all drivers and passengers, or even revise the traffic laws to regulate wearing seat belts. However, the physiological and mental performance is difficult to be constrained. Drivers who suffer from fatigue in long-distance driving would be more likely to get in serious accidents. Moreover, drivers with unobvious and unnoticed cardio- vascular disease (CVD) have higher risk of causing traffic accident during long-distance driving.

As one of the fatigue detection technologies, physiological features contribute significantly to fatigue recognition, such as electroencephalogram (EEG) data analyzed to obtain the variation of brain activity, electrocardiogram (ECG) data used to study heart rate variability (HRV) at progressive stages of fatigue [3-5]. Rogado et al. developed a driver fatigue detection system based on HRV from ECG during driving period [6]. However, challenge still remains in these methods that it is so difficult to acquire these physiological signals in a non-contact way and the driver will be distracted by measuring electrodes inevitably, because usually electrodes and wires need to be contacted with drivers directly. Moreover, due to various limitations of capacitive ECG measurement [7], only the surface skin of both hands can be contacted with dry electrodes to detect ECG signals without using any wires or $\mathrm{Ag} / \mathrm{AgCl}$ electrodes.

Ballistocardiography (BCG) is a noninvasive method to record the ballistic force caused by the motion of heart and blood during each cardiac cycle. BCG changes are different between subjects, which may reflect the short- or long-term cardiovascular state of individuals.

This work proposed a prototype of steering wheel cover to collect ECG signal. Since someone used to hold the steering wheel with only single hand during cruising, in order to ensure signal 
integrity and data continuity, a force sensor underneath the driver's seat was placed to simultaneously measure sitting BCG, so as to help us devising an effective monitoring system during long-distance driving.

This paper presents a particular implementation of a practical and energy-efficient long-term driving monitoring system consisting of three parts: 1) sensors integrated into seat cushion and steering wheel; 2) data collection and transmission system (DCTS) with analog front end (AFE); and 3) data communication protocol and interface. Additionally, four volunteers were measured in sitting positions to simulate the situation of smooth driving, and experimental results were discussed.

\section{Materials and Methods}

Dry Electrodes Using Conductive Foam. Traditionally, the standard $\mathrm{Ag} / \mathrm{AgCl}$ electrodes with wet conductive gels are used for ECG recordings. Since the gel can cause skin irritations and support bacterial growth, the disposable electrodes can be used for a few days only. Furthermore, dehydration of the hydro-gel modifies the electrode impedance, which generates noise and other artifacts [8].

In order to acquire ECG signal without making distraction to drivers, the soft dry electrodes created by conductive foam material were integrated into the steering wheel cover. As shown in Fig. 1 , left electrode area was contacted with palm of left arm (LA), while right one was divided into right arm (RA) and driven-right-leg (DRL) parts by insulated rubber. The size of the conductive foam was $2 \mathrm{~cm}$ in width and $10 \mathrm{~cm}$ in length.

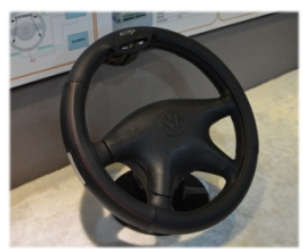

(a)
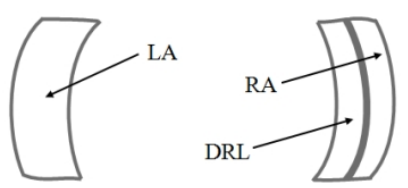

(b)

Fig. 1. (a) Photograph of the steering wheel cover. (b) Layout of the electrodes.

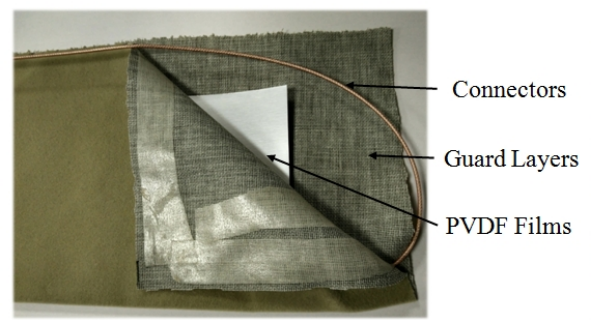

(a)

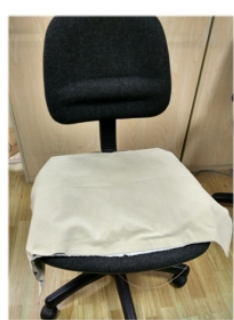

(b)

Fig. 2. (a) Inner structure of PVDF film sensor. (b) Implementation of seat cushion.

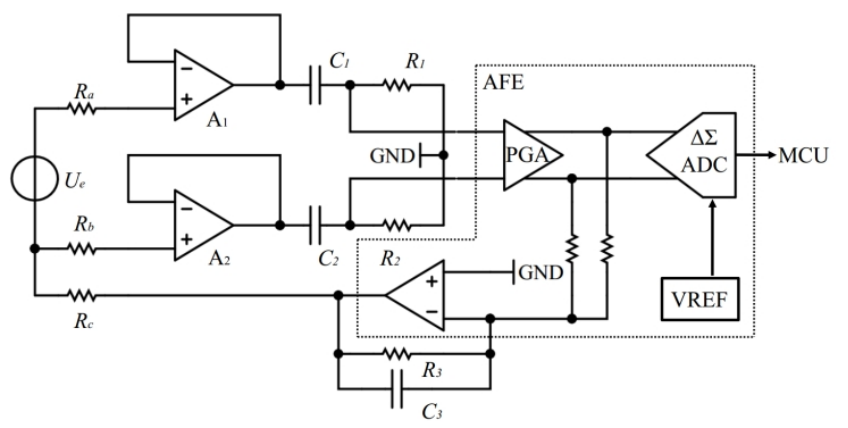

Fig. 3. Schematic circuits of ECG detection using foam electrodes. 


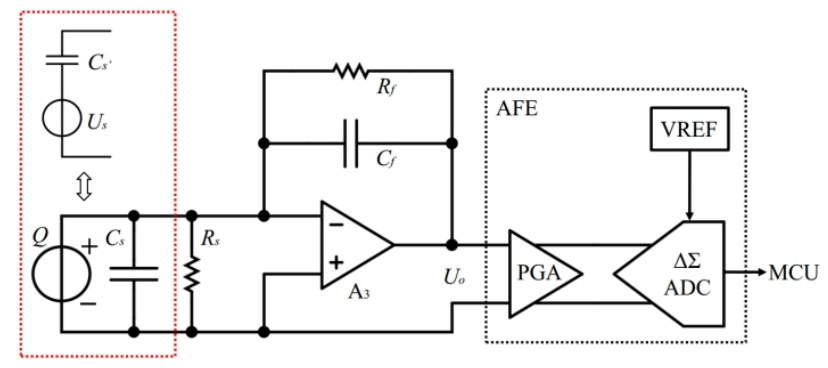

Fig. 4. Schematic circuits of BCG measurement based on PVDF film.

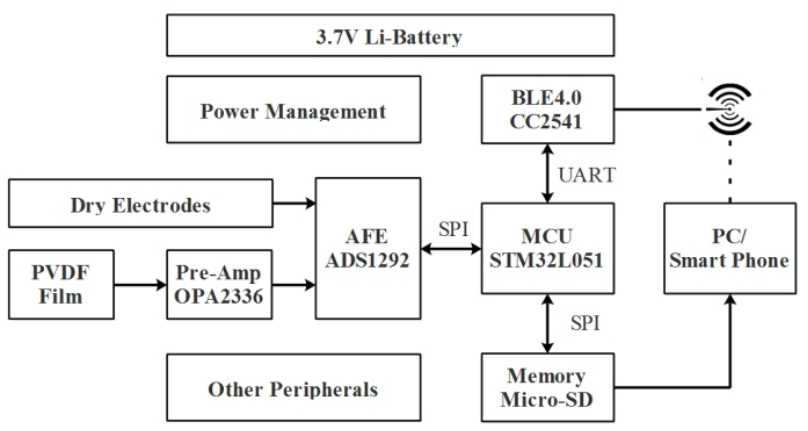

Fig. 5. Block diagram of proposed data collection and transmission system (DCTS).

Table 1. Symbols and significations in Fig. 3 and Fig. 4

\begin{tabular}{ll}
\hline \hline Symbol & Signification \\
\hline$R_{a}, R_{b}, R_{c}$ & Current-limiting resistance \\
$\mathrm{A}_{1}, \mathrm{~A}_{2}$ & Impedance matching pre-amplifier \\
$\mathrm{A}_{3}$ & Charge pre-amplifier \\
$R_{l}, C_{l}, R_{2}, C_{2}$ & Passive high-pass filter network \\
$Q$ & Charge generated by pressure changes \\
$C_{s}$ & Equivalent capacitance of sensor \\
$R_{s}$ & Insulation resistance of sensor \\
$C_{s}$ & PVDF output capacitance \\
$U_{s}$ & PVDF output signal voltage source \\
\hline \hline
\end{tabular}

A PVDF Piezoelectric Film Sensor. As a pressure-sensing element, PVDF polymer film is strained and due to its piezoelectric properties an electric charge is generated on the welding electrodes, when a pressure change is applied to the sensor. Like most other piezoelectric sensors, the PVDF film cannot measure static pressure because of slow discharging caused by its electrical resistance. Thus, the minimum cut-off frequency of pressure changes is about $0.01 \mathrm{~Hz}$, and the suggested response time of the sensor is less than $100 \mu$ s [9].

As illustrated in Fig. 2, 0.28mm thick PVDF film with double-sided metal coating is guarded by the layer of antistatic fabric. Shielded cable has contact with the welding wire, which is conductive. This PVDF film sensor $60 \mathrm{~cm}$ in length, $40 \mathrm{~cm}$ in width was placed under the thighs in the seat of office chair to acquire BCG signal under simulated driving environment.

Circuit Design and Data Collection. According to the electrical circuit theory, suitably increasing input impedance in analog front end (AFE) of dry-contact foam sensor is essential for good performance. A CMOS operational amplifier (OPA2336, Texas Instruments, Inc., Dallas, USA) was placed to match with the foam electrodes. Due to the random motion artifacts, baseline drift or low frequency interference can appear inevitably on the output of preamplifier. Therefore, a first-order passive high-pass filter $\left(f_{-3 d B}=1 \mathrm{~Hz}\right)$ was designed to decrease the low-frequency noise. Equivalent DRL circuits was applied to reduce common-mode interference using internal amplifier of AFE system (ADS1292, Texas Instruments, Inc., Dallas, USA), shown in Fig. 3. The symbols and significations are illustrated in Table 1.

Fig. 4 shows the schematic circuits used to measure small charge changes, which is produced by the PVDF film. The output voltage $\left(U_{o}\right)$ of the charge amplifier stage can be calculated by Eq. 1 . 


$$
U_{o}=-\frac{\frac{1}{j W c_{r}}}{\frac{1}{j W \tau_{s^{\prime}}}} U_{s}=-\frac{\tau_{s^{\prime}}}{\tau_{f}} U_{s}=-\frac{\tau_{s^{\prime}}}{\tau_{f}} \frac{Q}{\tau_{s^{\prime}}}=-\frac{Q}{\sigma_{f}}
$$

Where $C_{f}$ is the feedback capacitance of the low input bias current (1pA) preamplifier (OPA2336, Texas Instruments, Inc., Dallas, USA) and $R_{f}$ is the feedback resistance added to prevent signal saturation effect.

A passive one-pole low-pass $\mathrm{RC}$ filter $\left(f_{-3 d B}=150 \mathrm{~Hz}\right)$ was placed in series between the charge amplifier and the integrated AFE. The ADS1292 is a dual-channel, simultaneously sampling, 24-bit delta-sigma $(\Delta \Sigma)$ analog-to-digital converter (ADC) with integrated programmable gain amplifiers (PGAs). During the BCG recording, ECG signal is simultaneously measured from hands by the AFE circuits at the same sampling rate of $250 \mathrm{~Hz}$. The BCG and ECG data packages from ADC were collected by the microcontroller (MCU) at the end of each sample using the SPI bus.

Data Communication. The complete data collection and transmission system (DCTS) is shown in block diagram form in Fig. 5. A Li-Battery (dc-3.7V, 1000mAh) provided clean power to the DCTS. The digitized output of ADS1292 was connected to the input port of STM32L051 (ST Microelectronics, Geneva, Switzerland), and then the digitized signal was immediately stored into micro-SD card onboard with the secure digital input/output interface (SDIO). Meanwhile, the current data package can be wirelessly transmitted to the on-board computer or smart phone using low-energy Bluetooth (BLE) module, which was configured as invisible UART mode with the speed of 57,600 baud rate.

Table 2. Power consumption of three operation modes

\begin{tabular}{ccl}
\hline \hline Mode & Average Power Consumption & \multicolumn{1}{c}{ Lifetime } \\
\hline Standy & $0.07[\mathrm{~mW}]$ & $>19[$ Month] \\
CR & $1.54[\mathrm{~mW}]$ & $>18[$ Day $]$ \\
RTC & $22.1[\mathrm{~mW}]$ & $>28[$ Hour $]$ \\
\hline \hline
\end{tabular}

\section{Results and Discussion}

Power Consumption for DCTS. The system provided three modes, including the standby mode, continuous recording (CR) mode and real-time communication (RTC) mode. The last two modes were used to acquire signals for DCTS. Table 2 shows average power consumption and lifetime depending on the working mode.

After the system was powered up, MCU configured specific registers of all used peripherals and initialized them into low-power mode or closed the partial power switch directly. Then, the average power consumption of DCTS dropped to about $0.07 \mathrm{~mW}$, and the system entered into standby mode (S0) which was the original mode of the finite state machine (FSM). Using the different external interrupt (EXTI), MCU was waked up into CR mode or RTC mode. In these operation modes, MCU spent most of the time on sleep in order to save energy. As soon as the data-ready interrupt arose from AFE, MCU read the data package and push them into FIFO area. After that, the program pointer returned back to the main process, and saved data into SD memory or transmitted them to the host device by the BLE module, if the flag of buffer-full was checked true. The serial receive interruption (RINT) can be generated to switch operation states by BLE communication. 

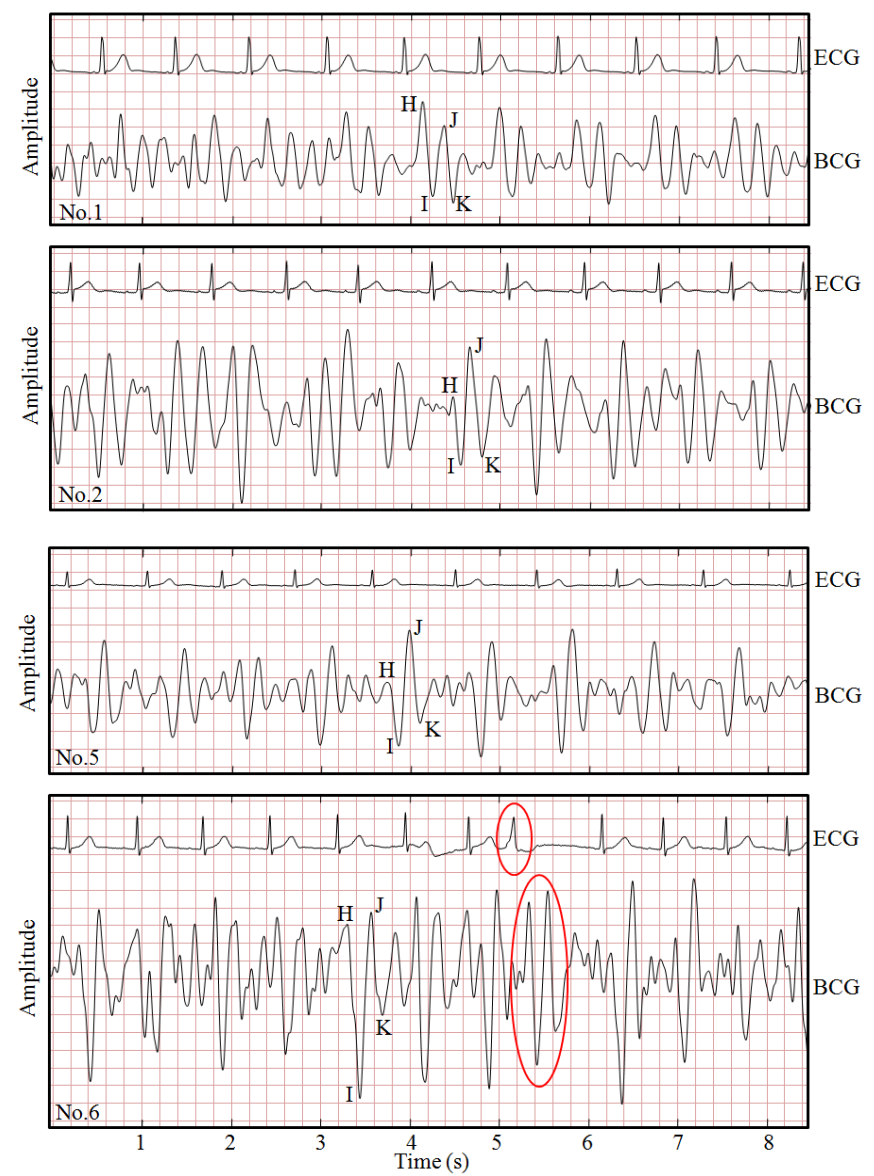

Fig. 6. Signals recorded in the sitting position from all subjects in the simulation experiments.

Example Waveforms From All Subjects. Four volunteers who had no history of cardiovascular disease were arranged for simulation experiments in the laboratory. The BCG and ECG signals were digitally Butterworth band-pass filtered in host computer with the following bandwidth, in hertz, respectively: 0.1 20 and 1 40.

Fig. 6 shows the recorded signals from these four subjects for a 8.5-s segment of the recording. All ECG signals appear to be of sufficiently high quality to readily detect the QRS complex, so as to identify the locations of the heartbeats. BCG $\mathbf{J}$ waves from all persons are clearly visible which describe the acceleration of blood in the descending and abdominal aorta, and the deceleration of blood in the ascending aorta [10]. A repeatable pattern of HIJK complex waves occurs with every heartbeat. Several researches have demonstrated that BCG signals can be used to calculate RJ interval (RJI), which is defined as the time interval from ECG R peak to BCG J peak, so as to realize the cuffless BP monitoring system [11].
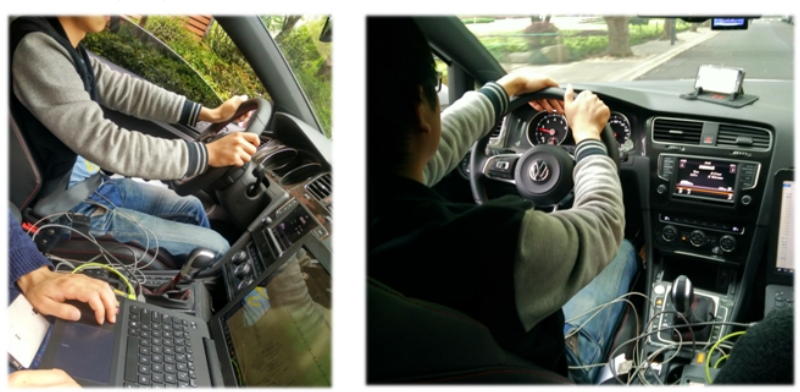

Fig. 7. Photographs of real vehicle driving experiment. 


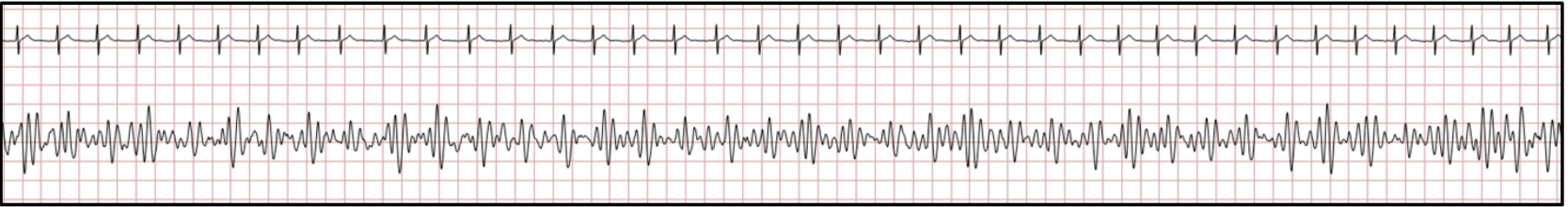

Fig. 8. Signals recorded during the highway driving.

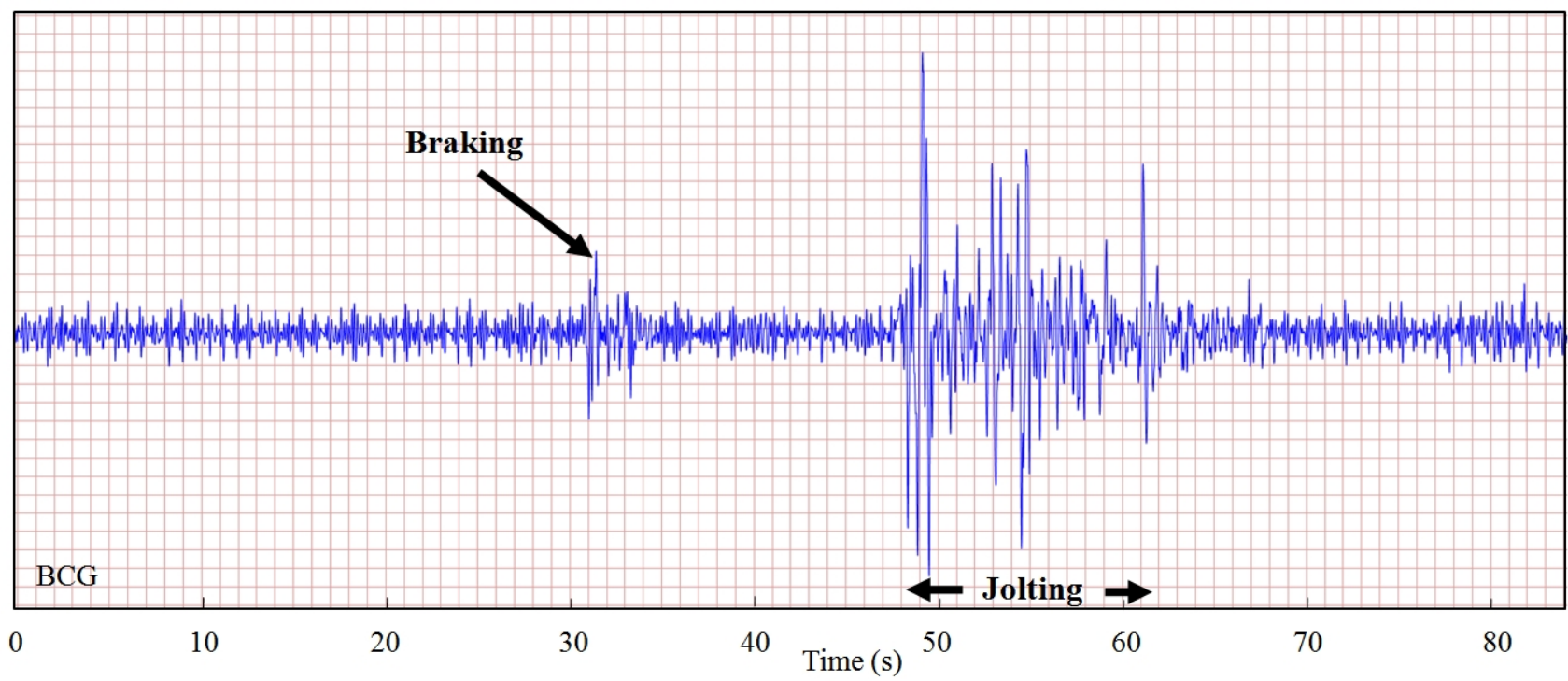

Fig. 9. 85-s BCG signal recorded from the volunteer who drove Golf car through the city roads.

A single ventricular premature contraction (VPC) is found in the last two signals from the fourth subject in Fig. 6. As the extension of driving time and distance, not only the brain but the cardiovascular system and muscles will be tied. The physiologic response to VPC's frequency may be related to driving fatigue.

Analysis and Discussion of Signals During Real Driving. Fig. 7 shows photographs of real car driving. A partner sitting beside the driver used the proposed DCTS to simultaneously acquire ECG and BCG signals. In order to collect complete data in various scenarios as much as possible, we drove through crowded city roads and expedite highways. Obviously, the highest frequency of fatigue and inattention occurs in the period of straight driving without swerve. Fig. 8 indicates a segment of recording during the highway driving, which demonstrates ECG and BCG signals of high quality. The ECG signal components such as Q, R, S and T waves can be identified clearly so as to increase the possibility of automatic morphology recognition by machine in the future. According to our investigation and statistics from the questionnaires, about $85 \%$ of 1,500 drivers tend to hold the steering wheel with left hand and right hand on the 8 to 10 o'clock and 2 to 4 o'clock direction respectively. Therefore, properly extending the length of foam electrode to cover more areas is beneficial to collect ECG signal by limb lead.

However, some drivers are accustomed to driving with only single hand sometimes which causes impossibility to acquire the ECG signal. In this particular case, BCG signal detection appears especially important for monitoring system.

As shown in Fig. 8 and Fig. 9, HIJK complex can be acquired steadily and clearly during except for speeding up, braking and jolting periods. Fortunately, the drivers tend to pay more attention to hold the steering wheel with both hands in unsteady conditions, thus the acquired ECG signals have satisfied performance. Due to the fast response of piezoelectric sensor using PVDF film, the duration of the motion artifacts is usually short enough. 


\section{Conclusions and Future Work}

Unlike other similar driving monitoring systems, the proposed implementation of DCTS provides fully unconstrained physiological signal acquisition during the period of long-term driving. By integrating the soft conductive foam and a PVDF film into steering wheel and driver's seat respectively, we can acquire ECG and BCG without any distraction to the drivers successfully and simultaneously. Our results demonstrate the practicability of proposed system for monitoring of health and fatigue in daily driving.

In the future work, we plan to improve stability and reliability of hardware system and focus on extracting the characteristic parameters to explore the statistical correlation between driving safety and physiological signals.

\section{References}

[1] Organization W H. Global status report on road safety - 2013: supporting a decade of action [J]. World Health Organization, 2013.

[2] $\mathrm{Chu} \mathrm{H}$ C. Assessing factors causing severe injuries in crashes of high-deck buses in long-distance driving on freeways [J]. Accident Analysis \& Prevention, 2014, 62(5):130-136.

[3] Lal S K L, Craig A, Boord P, et al. Development of an algorithm for an EEG-based driver fatigue countermeasure [J]. Journal of Safety Research, 2003, 34(3):321-8.

[4] Peter R, Zoltan H, Ivan P, et al. Measurement of mental fatigability by task related spectral EEG. A pilot study [J]. Ideggyógyászati Szemle, 2009, 62(1-2):36-40.

[5] Nayak B P, Routray A, Kar S, et al. A Biomedical Approach to Retrieve Information on Driver's Fatigue by Integrating EEG, ECG and Blood Biomarkers during Simulated Driving Session [C]. Intelligent Human Computer Interaction (IHCI), 2012 4th International Conference on, IEEE, 2013:1-6.

[6] Rogado E, Garcia J L, Barea R, et al. Driver fatigue detection system [C]. Proceedings of the 2008 IEEE International Conference on Robotics and Biomimetics. IEEE Computer Society, 2009:1105-1110.

[7] Chi Y M, Jung T P, Cauwenberghs G. Dry-contact and noncontact biopotential electrodes: methodological review [J]. IEEE Reviews in Biomedical Engineering, 2010, 3:106-119.

[8] Gruetzmann A, Hansen S, Müller J. Novel dry electrodes for ECG monitoring [J]. Physiological Measurement, 2007, 28(11):1375-90.

[9] Shirinov A V, Schomburg W K. Pressure sensor from a PVDF film [J]. Sensors \& Actuators A Physical, 2008, 142(1):48-55.

[10]Alametsä J, Viik J, Alakare J, et al. Ballistocardiography in sitting and horizontal positions [J]. Physiological Measurement, 2008, 29(9):1071-87.

[11]Shin J H, Lee K M, Park K S. Non-constrained monitoring of systolic blood pressure on a weighing scale [J]. Physiological Measurement, 2009, 30(7):679-693. 
